\title{
Harmonic and rhythmic influences on musical expectancy
}

\author{
MARK A. SCHMUCKLER \\ University of Toronto, Scarborough College, Scarborough, Ontario, Canada \\ and \\ MARILYN G. BOLTZ \\ Haverford College, Haverford, Pennsylvania
}

\begin{abstract}
The effects of harmony and rhythm on expectancy formation were studied in two experiments. In both studies, we generated musical passages consisting of a melodic line accompanied by four harmonic (chord) events. These sequences varied in their harmonic content, the rhythmic periodicity of the three context chords prior to the final chord, and the ending time of the final chord itself. In Experiment 1, listeners provided ratings for how well the final chord in a chord sequence fit their expectations for what was to come next; analyses revealed subtle changes in ratings as a function of both harmonic and rhythmic variation. Experiment 2 extended these results; listeners made a speeded reaction time judgment on whether the final chord of a sequence belonged with its set of context chords. Analysis of the reaction time data suggested that harmonic and rhythmic variation also influenced the speed of musical processing. These results are interpreted with reference to current models of music cognition, and they highlight the need for rhythmical weighting factors within the psychological representation of tonal/pitch information.
\end{abstract}

One basic concern of cognitive psychologists has been understanding the structure of cognitive representations. In audition, the two primary areas in which such investigations have proceeded involve examining the nature of listeners' representations of linguistic and musical structure. Music, in particular, provides an excellent domain for such inquiries; musical behavior requires a diversity of perceptual, cognitive, and motoric skills, making it a paradigmatic case of general psychological functioning. In audition, the availability of detailed theoretical analyses of musical structure provide an independent source of hypotheses and predictions concerning the perceptual and cognitive processing of music; such analyses have proven invaluable in our understanding of musical cognition.

As a possible consequence of these advantages, a number of informal and formal theoretical frameworks for understanding musical perception have been proposed by psychologists and musicologists (Bharucha, 1987a, 1987b, 1989; Deutsch, 1969; Deutsch \& Feroe, 1981; Dowling, 1978; Jones, 1976; Krumhansl, 1990; Krumhansl \& Kessler, 1982; Lerdahl \& Jackendoff, 1983; Meyer, 1956; Narmour, 1991a, 1991b). One such

This research was supported by a grant from the Natural Sciences and Engineering Research Council of Canada to the first author. The authors would like to thank Jim Carlsen and an anonymous reviewer for their comments on an earlier draft of this manuscript. Correspondence concerning this article should be sent to M. A. Schmuckler, Division of Life Sciences, University of Toronto, Scarborough Campus, Scarborough, ON, Canada, M1C 1A4 (e-mail: marksch@1ake.scar. utoronto.ca). formal model involves a neural net architecture, proposed by Bharucha and colleagues (Bharucha, 1987a, 1987b, 1989; Bharucha \& Stoeckig, 1986, 1987; Bharucha \& Todd, 1989), which models listeners' cognitive representations of musical pitch and complex pitch structures within meaningful musical contexts. Simply described, this model consists of a network of units that represent musically meaningful events. In this model, the lowest input level represents individual tones or pitches that contribute to a second level of major and minor chords (simultaneous sounding of tones). This "chord" level then connects with a "tonality" level, which represents the abstract, hierarchical relations existing between the tones and chords that characterize Western music. Connections between these levels are weighted so that activation at the tone level spreads differentially to chord units, which then differentially activate tonality units, and so on.

This model accounts for a diverse array of empirical findings in the musical cognition literature, including measures of the perceived relatedness among pairs of tones and chords (Bharucha \& Krumhansl, 1983; Krumhansl, 1979; Krumhansl \& Kessler, 1982), memory confusions (Bharucha \& Krumhansl, 1983; Cuddy, Cohen, \& Miller, 1979; Krumhansl, 1979; Krumhansl, Bharucha, \& Castellano, 1982), and response times to musical events (Bharucha, 1987b; Bharucha \& Stoeckig, 1986, 1987). One particularly compelling application of this model involves the concept of musical expectancy, or a listener's ability to anticipate, with varying degrees of specificity, upcoming musical events on the basis of previously heard musical events. An ex- 
ample of how Bharucha's (1987a, 1989) architecture captures the generation of musical expectancies has been revealed in studies of musical priming by Bharucha and colleagues (Bharucha \& Stoeckig, 1986, 1987; Tekman \& Bharucha, 1992). In these studies, listeners detected mistunings in the second of two sequential chords that were either harmonically related or unrelated. Results indicated that the processing of related chords occurred more quickly and accurately than the processing of unrelated chords, suggesting that the listeners generated expectations for the second chord on the basis of the preceding context chord. This pattern of priming is assumed to reflect the spread of activation among the chord units of Bharucha's model, based on the differentially weighted connections across related and unrelated note, chord, and tonality units.

On a general level, and as evidenced by the above model, the majority of theoretical frameworks for understanding musical cognition have treated the perception of pitch relationships separately from the perception of metrical and rhythmic structure, implicitly assuming these to be autonomous domains. Bharucha (1989), for example, is explicit about this division, citing evidence from neurological studies suggesting a dissociation of musical parameters in terms of hemispheric functioning. Additionally, evidence from some psychological studies indicates independent and additive processing of pitch and temporal information in various musical contexts, such as the judged completeness of musical phrases (Palmer \& Krumhansl, 1987a, $1987 \mathrm{~b}$ ) or the judged similarity of melodies (Monahan \& Carterette, 1985). As such, it is understandable that models of musical cognition have, by and large, focused on the processing of pitch or temporal components individually, resulting in models that capture one or the other aspect, but not both.

Intuitively, however, models of musical structure seem incomplete when such a strong dissociation between pitch and temporal components is assumed. There is, in fact, at least one theoretical approach that claims that the temporal and melodic information within a melody is not only structurally entwined, but also exerts an interactive influence on behavior. Both Jones and Boltz (Boltz \& Jones, 1986; Jones \& Boltz, 1989) have demonstrated that in the structural description of a melody, the interplay of meter and rhythm gives rise to temporal accents that "stand out" and capture one's attention. These accents may arise from pauses or prolonged durations, but in either case they tend to regularly recur in time and bear a lawful relationship to the underlying pitch organization of a tune. For example, temporal accents often coincide with contour peaks and reversals, as well as with large pitch intervals (Boltz \& Jones, 1986; Jones, 1987a, 1987b). In addition, tonic triad members marking phrase ending points are typically accentuated, which in turn provides momentary points of resolution within a melody and a reaffirmation of the underlying tonal scheme (Berry, 1976; Cone, 1968; Piston, 1978).
This joint relationship between temporal and pitch structure serves several important cognitive functions for a listener. Given that temporal accents tend to regularly recur in time and attentionally highlight the organization of pitch relationships, these can be used to reliably guide attending over the melody's time span, facilitating attentional tracking and perceptual learning (Boltz, 1991), while also yielding superior recall (Boltz, 1991; Boltz \& Jones, 1986; Deutsch, 1980) and recognition memory performance (Boltz, 1993; Jones, Boltz, $\&$ Kidd, 1982). More importantly, however, a regularly recurrent array of temporal/pitch accents allows listeners to extrapolate the melody's preceding context and generate expectancies about both the "what" and "when" of upcoming structural relationships. Temporal accents thereby support anticipatory attending, so that a listener's attention is directed toward particular melodic relations that occur at specific points in time.

This approach to music cognition envisions a joint relationship between temporal and pitch structure that is assumed to be reflected within cognitive performance. There are, in fact, several studies supporting this claim. One comes from a series of experiments by Boltz (1993), who investigated the types of structural relations that support the generation of musical expectancies. Using folk melodies as experimental stimuli, Boltz (1993) manipulated the pattern of temporal/melodic accentuation within a melody's preceding context so that expectancies, if generated, could carry over to a series of isochronous notes in the latter half of the melody. The ability to detect deviant pitch changes in the final variation was then evaluated. The results of both reaction time and accuracy data indicated that expectancy generation depended upon the interactive influence of temporal and melodic structure, so that expectancies did not independently arise from a consistent accentuation of meaningful tonal intervals (i.e., phrase ending points), nor from an invariant periodicity of temporal accentuation. Instead, both structural relations had to co-occur in order for anticipatory attending to emerge.

This joint relationship between temporal and melodic structure has also been observed in perceptual judgment tasks. For example, Boltz (1989a) asked musically sophisticated listeners to rate the degree of resolution in a set of folk melodies varying in their tonal ending and temporal accent structure. Melodies ending on the leading tone-to-tonic interval were judged as being the most complete, and melodies ending on the leading tone interval were judged as being the least complete. In addition, the temporal accent structure of a melody influenced ratings; the highest degree of resolution was observed for melodies ending "on time" through an invariant pattern of temporal/melodic accents. Accent structures that led to endings that occurred earlier or later than expected resulted in significantly lower resolution ratings. Subsequent studies have revealed that these effects also exert a significant impact on time estimation behavior. When listeners are asked to make relative or absolute time judgments, melodies appearing to 
end on time yield highly accurate estimates of the tunes' total duration. In contrast, melodies that violate one's expectancies by ending "too early" or "too late" produce under- and overestimates of melody duration, respectively (Boltz, 1989b; Jones, Boltz, \& Klein, 1993).

Taken together, these results indicate that the confirmation or violation of temporal expectancies significantly influences the ability to detect deviant pitch changes, judgments of melodic completion, and estimates of a melody's total time span. More generally, they illustrate that temporal and melodic relations influence both attention to and the processing of auditory and $\mathrm{mu}-$ sical events. One question to consider is whether temporal expectancies affect other aspects of musical listening and, if so, what the nature of this effect is. Unlike the melodies used in previous studies, most Western music displays a complex interweaving of melodic lines in which the unfolding array of tonal intervals in one register (treble or bass) complements those in another register. For example, a melody may display a melodic pattern in the upper treble clef that is accompanied by a particular sequence of chords in the bass. This, in turn, supports the generation of listeners' expectancies so that one can anticipate the melodic and harmonic content of upcoming tonal relations as well as when in time they will occur. The purpose of the present research was to investigate this issue by considering whether expectancies for certain chord sequences vary as a function of a passage's preceding temporal context. That is, is there an interaction between the confirmation/violation of temporally based expectancies and the instantiation of harmonic expectancies? If, in fact, such effects are observed, this would suggest that current models of music cognition need to acknowledge the role of temporal/ rhythmic factors in the mental representation of musical structure. This is particularly true of models that attempt to account for the formation of musical expectancies that are extrapolated from an underlying tonal system (Bharucha, 1987a, 1987b, 1989; Bharucha \& Todd, 1989).

\section{EXPERIMENT 1 Expectancy Ratings}

Expectancies concerning the upcoming "what" of a melody have been extensively investigated in previous psychological studies and incorporated into computer simulations of musical processing (Bharucha, 1987a, 1987b, 1989; Gjerdingen, 1989, 1990). Within the context of laboratory experimentation, expectancies have often been investigated with production tasks, in which subjects are presented with an initial melodic context and asked to predict a future interval through vocal (Carlsen, 1981; Unyk \& Carlsen, 1987) or keyboard responses (Abe \& Hoshino, 1990; Schmuckler, 1990). Carlsen (1981), for example, presented listeners with a set of two-tone pitch intervals and asked them to sing their continuations. Results revealed that small-interval continuations ( 3 semitones or fewer) were more likely to be produced than large ones, and that some melodic contexts produced stronger expectancies than others.

A series of studies by Schmuckler (1989) later converged on these findings by systematically assessing the impact of various music-theoretic predictions on expectancy generation. In contrast to two-tone context intervals, subjects were presented with a Schumann composition and, at various stopping points, were asked to rate how well a given continuation tone conformed to their expectancies. The overall pattern of results revealed that ratings varied as function of the implied tonal content of the passage (Krumhansl \& Kessler, 1982) and reflected certain melodic processes, such as continuation and reversal (Meyer, 1973; Narmour, 1989). In addition, a subsequent production task indicated that pianistperformed expectancies of melodic and combined melodic/ harmonic expectancies correlated with the earlier rating scale measures of expectancies (Schmuckler, 1989) and were predictable not only from models of tonal processing, but also from the preceding metrical structure of the piece (Schmuckler, 1990).

The present set of experiments extends this previous research by assessing the potential joint impact of harmonic and rhythmic context on the formation of expectancies. In both studies, subjects were presented with a series of musical passages containing a melodic line in the upper (treble) register and a set of four accompanying chords in the bass. These chord sequences represented different types of cadences commonly observed in Western music. In each, the final chord of the progression was one that was strongly expected, moderately expected, or harmonically unexpected. In addition, the temporal structure of these passages was manipulated along two different dimensions. One involved the temporal periodicity of the chords themselves. In some versions of the passages, the initial three context chords of a progression invariantly occurred on the first beat of a measure, thereby generating the expectancy that the fourth and final chord would appear with this same periodicity. In other versions of the sequences, the temporal periodicity between successive chords varied such that listeners were unable to anticipate when the next chord would occur. The second manipulation involved the relative timing of the final chord itself. In some experimental conditions, the fourth chord appeared on the first beat of the last measure and was therefore temporally predictable (i.e., on time) when the preceding context chords were invariantly timed. In other conditions, however, the final chord appeared one beat earlier or later than expected, so that listeners' temporal expectancies were violated.

These manipulations of temporal and harmonic structure allow one to assess several different views of music cognition. On the one hand, it is possible that expectancy formation is relatively impervious to rhythmic manipulation, so that one's expectations for a single harmonic event do not change in the face of temporal variation. Psychological models of musical processing that focus on pitch information (Bharucha, 1987a, 1987b, 1989) 
might be construed as making such a prediction. In contrast, rhythmic variation might significantly affect expectancy formation, so that judgments of continuations vary not only as a function of the harmonic content of these events, but also in response to the temporal context. Although the previously discussed models of musical processing do not explicitly represent rhythmic context, in principle nothing in these models is anathemic to such rhythmic effects.

If rhythmic context does influence expectancy formation, then a secondary goal of this experiment involves assessing the nature of this influence. One possibility is that rhythmic context impacts dramatically on expectancy formation, so that an unexpected event given one rhythmic context becomes a highly expected event given a second rhythmic context, even though the harmonic information remains constant. On the other hand, rhythmic context might have a more subtle influence on expectancy formation, resulting in a systematic variation of perceived expectancies in accordance with changing context, while at the same time retaining distinctions between strongly expected and unexpected events. The implications of these two different patterns of results will be elaborated on later in this article.

\section{Method}

Subjects. Thirty-two listeners participated in this experiment, receiving either extra credit in an introductory psychology course at the University of Toronto, Scarborough Campus, or monetary compensation of $\$ 7.00$. All the subjects reported normal hearing, and had completed at least 5 years of musical training.

Apparatus. All stimuli were generated by a Yamaha DX7 synthesizer controlled by an IBM PC, using a Roland MPU-401 Midi interface. All sequences were recorded onto cassette audiotape and presented to the listeners via a Technics RS-M7 cassette deck. Then they were fed through a Mackie 1202 mixer and presented to the listeners over two Boss MA-12V micro monitors. The timbre of all melodies approximated that of a celeste.

Stimulus materials. Excerpts from 12 songs were selected and adapted from a music composition book. ${ }^{1}$ All contained four measures conforming to a $4 / 4$ meter wherein the melodic line always occurred in the upper register, with a harmonic accompaniment of four chords in the bass. Each of these accompaniments represented one of three common chord cadences: an "authentic" cadence (roman number notation: vi-IV-V-I), a "half" cadence (iii-vi-ii-V), or a "deceptive" cadence (IV-I-V-vi). Melodies were sometimes modified slightly to better fit these underlying chordal sequences. Of the 12 melodies, 4 were accompanied by the authentic cadence, 4 by the half cadence, and 4 by the deceptive cadence. Within each chord cadence, each of the 4 melodies differed in its underlying tonality and was based on the C, D, F, or G major diatonic scales.

For all stimuli, tone duration (on time) and intertone interval (off time) were held constant with the following values-whole notes $=1,600 \mathrm{msec}$ on, $800 \mathrm{msec}$ off; half notes $=800 \mathrm{msec}$ on, $400 \mathrm{msec}$ off; quarter notes $=400 \mathrm{msec}$ on, $200 \mathrm{msec}$ off; dotted eighth notes $=300 \mathrm{msec}$ on, $200 \mathrm{msec}$ off; 8 th notes $=200 \mathrm{msec}$ on, $100 \mathrm{msec}$ off; and 16 th notes $=100 \mathrm{msec}$ on, $50 \mathrm{msec}$ off.

These songs were then manipulated along three different dimensions; Figure 1 shows a sample of one of the melodies, along with the various manipulations described below. One manipulation involved the harmonic identity of the final chord in the sequence; this chord varied in terms of its perceived expectancy strength. One ending consisted of a high-expectancy chord, or the chord tra- ditionally completing the musical cadence, as described above (e.g., the half cadence ending with the $\mathrm{V}$ chord). A second ending consisted of a medium-expectancy, or a harmonically related chord. The determination of medium expectancy was based on Bharucha and Krumhansl's (1983) data concerning the perceived relations between chords within a tonal context, as well as Piston's (1978) music-theoretic predictions of common harmonic progressions; these quantifications are reasonable predictors of harmonic expectancy formation (Schmuckler, 1989). Finally, a third ending consisted of a low-expectancy chord, drawn from outside the key of the passage.

A second manipulation of these sequences involved the temporal periodicity of chord onsets for the first three chords of a cadence. In one version, the chord progression had an invariant periodicity; chord onsets always occurred on the first beat of the first three measures. In a second version, the chord progression had a variant periodicity; chord onsets coincided with the second, third, or fourth beats of the first three measures. ${ }^{2}$

A final manipulation involved the ending time of the fourth and final chord in the last measure of each sequence. In the "on-time" version, the onset of the fourth chord coincided with the first beat of the fourth measure and was temporally predictable from the preceding chords when invariantly timed. In the "early" version, the fourth chord occurred on the fourth beat of the third measure; in this case, the chord occurred early, relative to the chord onsets in the invariant periodicity condition. In the "late" version, the fourth chord was heard on the second beat of the fourth measure and was late, relative to the chord onsets of the invariant periodicity condition.

Design and conditions. Crossing the manipulations of harmonic identity (three levels), temporal periodicity (two levels), and ending time (three levels) produced 18 possible versions for each of the 12 songs, yielding a total of 216 experimental patterns; nested within these 12 songs was the additional factor of chord cadence (three levels). These experimental patterns were randomized into four sets of 72 sequences. Within each set of 72 passages, 36 had an invariant periodicity, and 36 had a variant periodicity. Within each set of 36 sequences, 12 ended with a highexpectancy chord, 12 with a medium-expectancy chord, and 12 with a low-expectancy chord. Within each set of 12 sequences, 6 of the passages ( 2 for each chord progression) had ending times in which the final chord occurred early or on time, whereas 6 had the final chord occurring on time or late. The assignment of specific passages to invariant versus variant periodicity and early/on time versus on time/late ending time was counterbalanced across the four sets of 72 patterns. Two different random orders were created for each set of 72 passages, providing two replications of each experimental pattern.

Procedure. The listeners were told that they would be participating in a study investigating judgments of chord progressions. They were told that on each trial, they would hear a high-pitched warning signal, followed $1 \mathrm{sec}$ later by a melody accompanied by a sequence of four chords in the bass clef. Their task was to decide how well the fourth and final chord "fit" with what they expected to occur at that point, on the basis of the preceding three chords. The listeners rated this chord on a 7-point scale $(1=$ chord is very unexpected or inappropriate; $7=$ chord is very expected or appropriate). The listeners had $4 \mathrm{sec}$ to record their response on an answer sheet, at which point the warning tone recurred, signaling the start of the next trial.

The listeners were assigned to one of four sets of counterbalanced stimuli and were run in groups of 1 to 4 . At the beginning of each session, they received six practice trials to familiarize them with the experimental procedure and the use of the rating scale. After these practice trials, they listened to the first block of 72 trials, took a short break, and then listened to the second block of 72 trials. The entire experimental session lasted approximately $1.25 \mathrm{~h}$ and was run in a single day. 


\section{Chord Cadence: iii - vi - ii - V}

\section{High Expectancy}

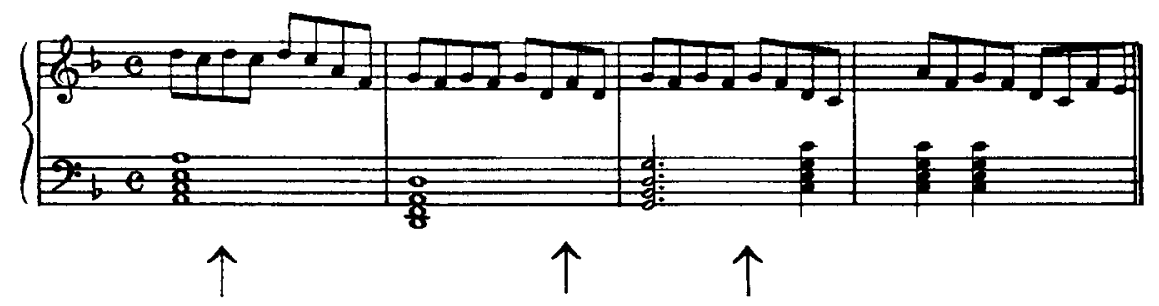

\section{Medium Expectancy}

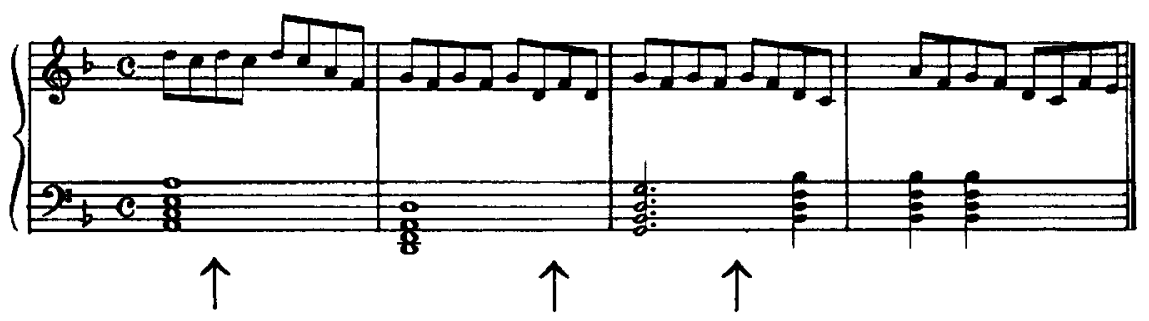

\section{Low Expectancy}

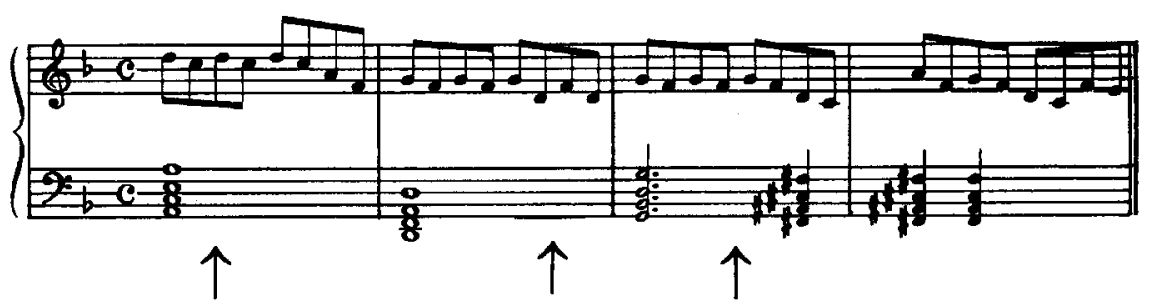

Figure 1. Example stimuli from Experiments 1 and 2, containing the different manipulations of harmonic identity, temporal periodicity, and ending time. Chord onsets in the imvariant condition occurred as shown in the musical score; those for the variant condition are notated by arrows underneath the musical staves. (Although shown as quarter notes, the final chord, shown in the earty, on-time, and late-onset conditions, had a duration similar to that of the preceding chords, as described in the text.)

\section{Results}

Prior to analysis, the listeners' ratings were averaged across the two replications of each passage in each condition and the four individual melodies comprising each chord progression. To assess intersubject reliability, ratings for the individual listeners were intercorrelated. The mean intersubject correlation was $.56(p<.01)$, with a range from -.25 to .94 . Other analyses examined whether the ratings differed as a function of the counterbalanced groups to which the listeners were assigned. An analysis of variance (ANOVA) failed to reveal a significant effect of this factor $\left[F(3,28)=0.94, M S_{\mathrm{e}}=6.93\right]$, and there were no interpretable interactions between this and other factors. As such, expectancy ratings were averaged across these groups for all subsequent analyses.
The principal analysis of interest was that examining expectancy ratings, using a four-way, within-subject ANOVA with the factors of chord cadence (three levels), harmonic identity (three levels), temporal periodicity (two levels), and ending time (three levels). Our results can be summarized as follows. Manipulating the harmonic identity of the final chord dramatically affected the listeners' judgments of this chord; high-, medium-, and low-expectancy chords received high-, moderate-, and low-expectancy ratings, respectively. Of more unique interest is the evidence suggesting that rhythmic variation (e.g., temporal periodicity and ending time factors) similarly influenced expectancy ratings. This effect is evident from the general difference in expectancy ratings for early versus on-time versus late final chords, as 
well as from a series of interactions between the rhythmic variation variables and harmonic identity.

In keeping with this summary, the results of the fourway ANOVA revealed the following effects. First, highexpectancy chords were rated as more appropriate to the musical passages than medium- and low-expectancy chords, producing a significant main effect for harmonic identity $\left[F(2,62)=173.55, M S_{\mathrm{e}}=8.74, p<.002\right]$. Second, early versus on-time versus late timing of these chords resulted in a small but significant main effect for ending time $\left[F(2,62)=5.69, M S_{\mathrm{e}}=2.21, p<.01\right]$, and significant two-way interactions between ending time and harmonic identity $\left[F(4,124)=8.14, M S_{\mathrm{e}}=1.08\right.$, $p<.001]$ and ending time and temporal periodicity $\left[F(2,62)=5.69, M S_{\mathrm{e}}=1.34, p<.01\right]$.

The most important finding of this study, however, was the significant three-way interaction between temporal periodicity, harmonic identity, and ending time $\left[F(4,124)=3.11, M S_{\mathrm{e}}=0.86, p<.02\right]$. This interaction, shown in Figure 2, averaged across the three different chord cadences, indicates that the listeners' perceptions of the appropriateness of a final harmonic event were a function not only of the harmonic identity of that event but also of the rhythmic context in which that event occurred. A series of Dunn comparisons revealed that when the unfolding chord context displayed an invariant periodicity, the perceived expectancy of the final chord varied as a function of when in time it occurred.

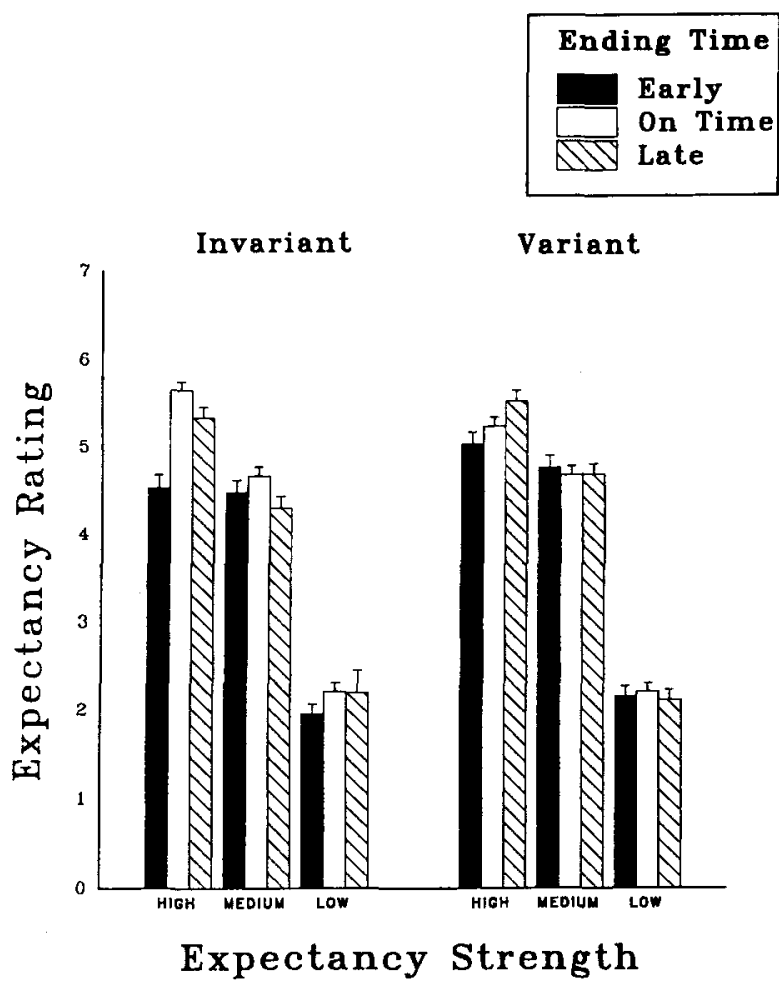

Figure 2. The mean expectancy ratings of Experiment 1, as a function of harmonic identity, temporal periodicity, and ending time. These ratings are averaged across the factor of chord cadence.
In particular, early endings were judged as significantly less appropriate (i.e., unexpected) than the chords that occurred either on time or late $(p<.05)$. Moreover, this effect was specific to high-expectancy chords and was not observed for medium- or low-expectancy chords. In contrast, when the preceding chord context had a variant timing, there was no systematic influence on expectancy ratings as a function of the temporal periodicity of the final chord.

Finally, two other significant three-way interactions were observed. These involved interactions between chord cadence, temporal periodicity, and ending time $[F(4,124)$ $\left.=3.62, M S_{\mathrm{e}}=1.10, p<.01\right]$, and between chord cadence, harmonic identity, and ending time $[F(8,248)=$ $\left.3.58, M S_{\mathrm{e}}=0.81, p<.002\right]$. Together, these findings indicate that the pattern of results observed in Figure 2 generalized to the half (iii-vi-ii-V) and deceptive (IV-I-V-vi) cadences; invariantly timed, high-expectancy chords were judged as more appropriate to a melody when the final chord of a sequence contained an on-time or late ending, relative to an early ending. In contrast, the differences between late and on-time endings were altered for full cadences (vi-IV-V-I) so that late final chords received slightly higher expectancy ratings than on-time final chords. In addition, the ratings for early chords were somewhat reduced. One possibility is that the sense of completion conveyed by the full cadence overrides, to some extent, the more subtle influences of the temporal factors. This effect is not surprising, given that the full cadence structure is considered by many music theorists as one of the most basic, underlying structures of Western tonal music (see Lerdahl \& Jackendoff, 1983, for examples).

Although the overall effects of this experiment have been attributed to manipulations of harmonic and rhythmic factors, varying the timing of the chord sequences relative to a constant melody also means that different melody tones were heard simultaneously with the chords across the different rhythmic conditions. One method for examining this potential confound involves using previously reported ratings on the perceived stability of individual notes within a tonal context (Krumhansl \& Kessler, 1982; Krumhansl \& Shepard, 1979). Simply described, Krumhansl and Kessler found that tones considered to be crucial from a music-theoretic view were perceived by listeners to "fit well" within the musical context, thereby receiving relatively high goodness-offit ratings. In contrast, tones that were theoretically unimportant received relatively low goodness-of-fit ratings. The average ratings for the 12 tones of the chromatic scale within a musical context have been referred to as a tonal hierarchy (Krumhansl, 1990), and they can be used as a quantitative measure for the melody tones contained in the sequences of the present experiment.

One such analysis examined whether the melody tone occurring simultaneously with the context chords systematically differed with the temporal periodicity of the chord context. An overall ANOVA revealed that 
this was not the case $[F(1,11)=0.74]$, suggesting that the invariant and variant timing conditions did not differ in terms of the perceived importance of the melody tone coincident with the context chords. A similar analysis examined whether the melody tone occurring simultaneously with the final chord differed as a function of ending time (early vs. on-time vs. late). This too failed to reveal any systematic effects $[F(1,11)=$ 0.29].

Using previously reported psychological data, the preceding analyses failed to show any relation between the hierarchical ranking of melody notes and the factors of temporal periodicity and ending time. A second possibility is that melody tones contained within the simultaneously sounding chord might be considered more consonant (i.e., "fit in" better) with this chord than melody notes not contained in the chord; this fit might therefore result in higher ratings for those chords. To explore this possibility, a series of ANOVAs analogous to those just described were conducted, using a count of the number of times that the melody note was contained within the simultaneously sounding chord as the dependent measure. The first analysis examined the influence of temporal periodicity (invariant vs. variant), and it failed to reveal any differences of this factor $[F(1,11)=0.19]$. A second analysis examined the number of times the melody tone occurred in the final chord as a function of the ending time factor (early vs. on-time vs. late); this analysis also failed to reveal any significant differences $[F(2,22)=0.62] .^{3}$ Overall, then, these analyses suggest that the observed effects on perceived expectancy were unbiased by the nature of the melodic material itself. 4

\section{Discussion}

The results of this study confirm our earlier intuitions that the formation of expectancies is simultaneously influenced by the harmonic and rhythmic information of a musical context. Not surprisingly, there was a cogent effect of the harmonic material, in keeping with predictions from music-theoretic sources (Piston, 1978) and previously reported psychological data (Bharucha \& Krumhansl, 1983; Schmuckler, 1989), in that chords typically expected to occur at the end of a given harmonic progression were, in fact, anticipated by the listeners. In contrast, chords that would not be expected to fit one's expectations for upcoming events were not, in general, anticipated by the listeners.

What is new in this study are the results suggesting that temporal factors also have an impact on expectancy formation for chord cadences. Overall, they indicate that a regularly recurrent sequence of chords establishes temporal expectancies that, when confirmed (i.e., are on time), enhance the harmonic appropriateness of chords when they themselves are strongly expected. However, these same chords can also appear less appropriate to a melody when their final onset violates temporal expectancies by occurring too early in time.

\section{EXPERIMENT 2 Reaction Time Effects}

Experiment 1 demonstrated that rhythmic context influenced expectancy formation. If these results are valid, they should generalize to an experimental context that relies on a different dependent measure of behavior. One such measure is reaction time, which is assumed to reflect the extent to which listeners are primed for the processing of musical materials (see Bharucha \& Stoeckig, 1986, 1987; Schmuckler, 1990). Moreover, there is some suggestion (Bharucha, 1987a; Bharucha \& Stoeckig, $1986,1987)$ that reaction time is a more sensitive measure of musical expectancy, given that reaction time is relatively impervious to explicit influences of theoretical knowledge of musical structure. Such knowledge could conceivably play a role in a rating scale task, particularly when subjects are musically sophisticated, as they were in Experiment 1.

There is ample evidence demonstrating that the processing of musical material is influenced by listenergenerated expectancies. Bharucha and colleagues (Bharucha \& Stoeckig, 1986, 1987; Tekman \& Bharucha, 1992) have provided the most elegant experimental evidence of this effect. In this work, which was briefly described previously, listeners detected mistunings of a target chord preceded by a prime chord. These authors observed a priming effect, in which listeners responded more quickly and accurately to target chords preceded by a musically related prime chord than to those preceded by a musically unrelated prime. One interpretation of these results is that prime chords engender expectancies in listeners for musically related material, thereby facilitating the processing of related material.

Given the results of Experiment 1, it is reasonable to ask whether, along with influencing listeners' ratings of the appropriateness of harmonic continuations, rhythmic context similarly affects reaction time to these same events. To answer this question, in Experiment 2 we combined aspects of the methodology of Experiment 1 with the priming paradigm used by Bharucha. Specifically, listeners made a yes/no judgment, as quickly as possible, on the harmonic appropriateness of a final harmonic event relative to its sequence of three context chords. Although this procedure does not produce data analyzable in terms of correct and incorrect responses, it has the advantage of allowing one to assess whether the reaction time data converge with the results of Experiment 1 .

\section{Method}

Subjects. Thirty-two listeners from an introductory psychology course at Haverford College participated in this experiment for course credit. All the subjects reported normal hearing, and they had completed at least 5 years of musical training.

Apparatus. All stimuli were constructed and generated with the MIDILAB software system (Todd, Boltz, \& Jones, 1989). During each experimental session, stimuli were presented on line by a Yamaha TX81Z FM tone generator controlled by an IBM AT 
computer with a Roland MPU-401 MIDI interface. Sequences of tones were amplified by a Kenwood KR-4010 receiver and played over Koss-Pro 4AAA Plus headphones at a comfortable listening level.

Procedure. Recorded instructions informed the listeners of pattern presentation details and task requirements. On each trial, a 1-sec warning tone preceded the presentation of a melody by $2 \mathrm{sec}$. During the 5 -sec response period that followed, the listeners decided whether the fourth and final chord accompanying the melody sounded appropriate and seemed to belong with the three preceding ones. They indicated their judgments on a response console by pressing the left or right buttons marked "belong" and "not belong," respectively. The listeners were asked to make this response as quickly as possible, even if the notes in the melody line were still playing. The computer automatically recorded the reaction time and belong/not-belong judgment on each trial.

The listeners were tested in small groups of 2-4 individuals. Each experimental session was approximately $1 \mathrm{~h}$ in duration, with a 5 -min rest break after the first 36 trials. The study was conducted over the course of 2 days; counterbalance order was blocked by day. Before each daily session, the subjects received 6 practice trials before beginning the experimental trials.

\section{Results and Discussion}

Experiment 2 resulted in two dependent measures. The first involves the percentage of times the listeners responded "belong" or "not belong" to the various sequences. This percent belong/not-belong judgment provides a replication for Experiment 1, in that sequences receiving high ratings in Experiment 1 should receive a high percentage of "belong" ratings in Experiment 2. Similarly, sequences receiving low-expectancy ratings should result in relatively few "belong" ratings (and correspondingly, many "not belong" ratings). The second dependent measure involves the reaction time required to produce either the "belong" or "not belong" judgment. This measure extends the results of Experiment 1 by assessing the processing speed of musical material as a function of harmonic and rhythmic context. For both dependent measures, prior to initial analyses, data were averaged across the four passages comprising each chord cadence and the two replications of each sequence in each condition.

Percent "belong" judgments. To assess intersubject reliability, the percentages of "belong" responses were intercorrelated. Across the four groups, the mean intersubject correlation was .74 $(p<.01)$, with a range from .40 to .93 . As with Experiment 1, initial analyses examined whether the percent "belong" ratings differed as a function of the counterbalanced groups to which the listeners were assigned. An ANOVA failed to reveal any significant effect of this factor $[F(3,28)=1.04]$, and there were no interpretable interactions between this and other factors. As such, the percent "belong" judgments were averaged across these groups for all subsequent analyses.

The next analysis examined the percent "belong" judgments with a four-way ANOVA, using the same four factors (chord cadence, harmonic identity, temporal periodicity, and ending time) that were used in Experiment 1 . Overall, the results of Experiment 2 closely converged with those of Experiment 1. Manipulating the harmonic content of the final chord produced a corresponding variation in whether the listeners rated this final chord as belonging to a given sequence. Highexpectancy chords received a higher percentage of "belong" ratings than did medium- and low-expectancy chords. Moreover, rhythmic variation (e.g., temporal periodicity and ending time) once again influenced the listeners' expectancies, producing interactions between the rhythmic and harmonic variations.

In support of these summarized results, there was a significant main effect for harmonic identity $[F(2,62)=$ $\left.1160.6, M S_{\mathrm{e}}=983.22, p<.001\right]$ that was qualified by the temporal context in which the sequence of chords appeared. This was demonstrated by significant twoway interactions between ending time and harmonic identity $\left[F(4,124)=5.83, M S_{\mathrm{e}}=480.14, p<.001\right]$, ending time and temporal periodicity $[F(2,62)=7.68$, $\left.M S_{\mathrm{e}}=511.69, p<.002\right]$, and chord cadence and harmonic identity $\left[F(4,124)=7.74, M S_{\mathrm{e}}=568.92, p=.001\right]$.

Of critical importance, however, is the evidence suggesting that harmonic appropriateness was simultaneously influenced by both harmonic and rhythmic factors; this result is demonstrated by the significant three-way interaction between temporal periodicity, harmonic identity, and ending time $\left[F(4,124)=3.31, M S_{\mathrm{e}}=\right.$ $400.8, p<.05]$, which appears in Figure 3 for these data averaged across the different chord cadences. Overall, it indicates that when high-expectancy chords unfolded with an invariant periodicity, they were judged as most

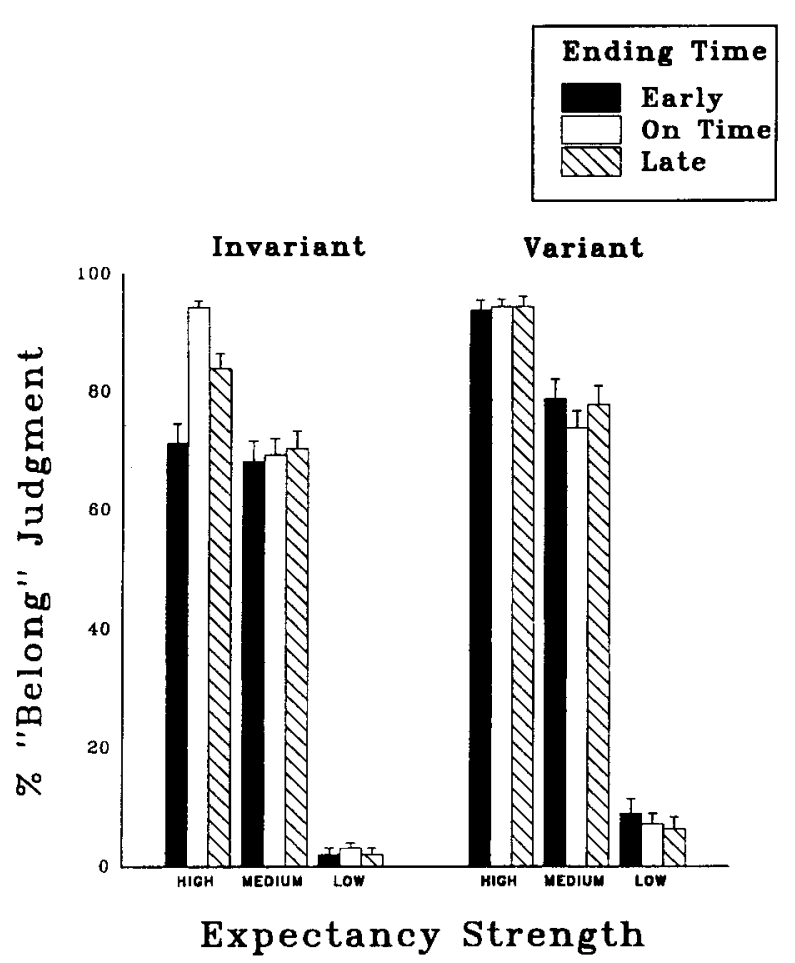

Figure 3. The mean percent "belong" judgments of Experiment 2 as a function of harmonic identity, temporal periodicity, and ending time. These percentages are averaged across the factor of chord cadence. 
appropriate to a melody when they ended on time. Late endings were rated as less appropriate, and early endings as the most inappropriate of all. Conversely, the timing of the final chord had no impact on "belong" judgments when a chord sequence was of medium or low expectancy.

By and large, the results of the percent "belong" judgments mimic the expectancy ratings gathered in Experiment 1 , albeit with some minor differences. To further compare the data of these experiments, the mean expectancy ratings from Experiment 1 were correlated with the mean percent "belong" judgments of Experiment 2 . This correlation was quite strong $[r(52)=.97$, $p<.001$ ], indicating a high degree of similarity between these two sets of data. Because of this, no further analysis of the percent "belong" judgments was performed. Subsequent analyses focused instead on the reaction time data.

Reaction time. Inspection of the percent "belong" data revealed that the listeners consistently used the "belong" and "not belong" labels with reference to the different levels of harmonic identity. That is, when the final chord was a high-expectancy chord, they invariably responded "belong"; when the chord was a lowexpectancy chord, they responded "not belong." Although the medium-expectancy chords did, in fact, fall between these two extremes, there remained a strong tendency for the listeners to respond "belong" (see Figure 3). On the basis of these rather dominant response tendencies, it was considered problematic to analyze reaction time data for both forms of response (i.e., "belong" and "not belong" results) for the different levels of harmonic identity; instead, only the reaction times for the primary response type were included in subsequent analyses. Assuming that a response of "belong" is, in fact, appropriate for high- and medium-expectancy endings (both endings do, after all, fall within the tonal framework of the passages) and that a response of "not belong" is appropriate for low-expectancy endings (given that they are outside the tonality of the passage), restricting analysis of reaction times to these response types is conceptually akin to analyzing only "correct" responses while removing reaction times for "incorrect" responses; this is a common procedure in the analysis of reaction time data. Correspondingly, subsequent analyses were conducted only on these data. ${ }^{5}$

As with the previous two analyses, an ANOVA was computed to assess whether reaction times differed as a function of the counterbalanced groups to which the listeners were assigned. In contrast to the previous two analyses, there was a significant effect of this factor $[F(3,28)=5.5, p<.01]$; the reaction times of the listeners in the fourth group were slower than those of the other three groups. However, given that this result generally did not interact with the main manipulations of this experiment, all data were averaged across this factor.

Again, the principal analysis of the reaction time data involved a four-way ANOVA, using the same factors that were used for the rating scale and percent "belong" data.
As before, there were differences in reaction time as a function of the harmonic appropriateness of the final event, as well as a general effect for the timing of the final chord. Interestingly, and in contrast to the percent "belong" and rating scale data, these effects were independent, with no observable interaction between the harmonic and rhythmic factors.

Statistical tests of these data support this descriptive pattern of results. First, there was a significant main effect for harmonic identity $\left[F(2,62)=58.95, M S_{\mathrm{e}}=\right.$ $453,821.27, p<.001]$. A series of Bonferroni post hoc comparisons indicated that reaction times to mediumexpectancy events exceeded both high- and lowexpectancy endings, and that reaction times to highexpectancy events were longer than those for low-expectancy events (all $p s<.01$ ). Second, there was a significant main effect for ending time $[F(2,62)=$ $\left.13.96, M S_{\mathrm{e}}=412,709.3, p<.001\right]$ wherein the reaction times to early endings were significantly longer than those for both on-time and late endings (all $p s<.01$ ). Finally (and again in contrast to the rating and percent "belong" judgments), there was no interaction between temporal periodicity, harmonic identity, and ending time $[F(4,124)=1.15]$. Figure 4 displays the reaction time data as a function of these three factors, averaged across the different chord cadences. 6

In sum, the present results converge with those of Experiment 1 by revealing that judgments of harmonic con-

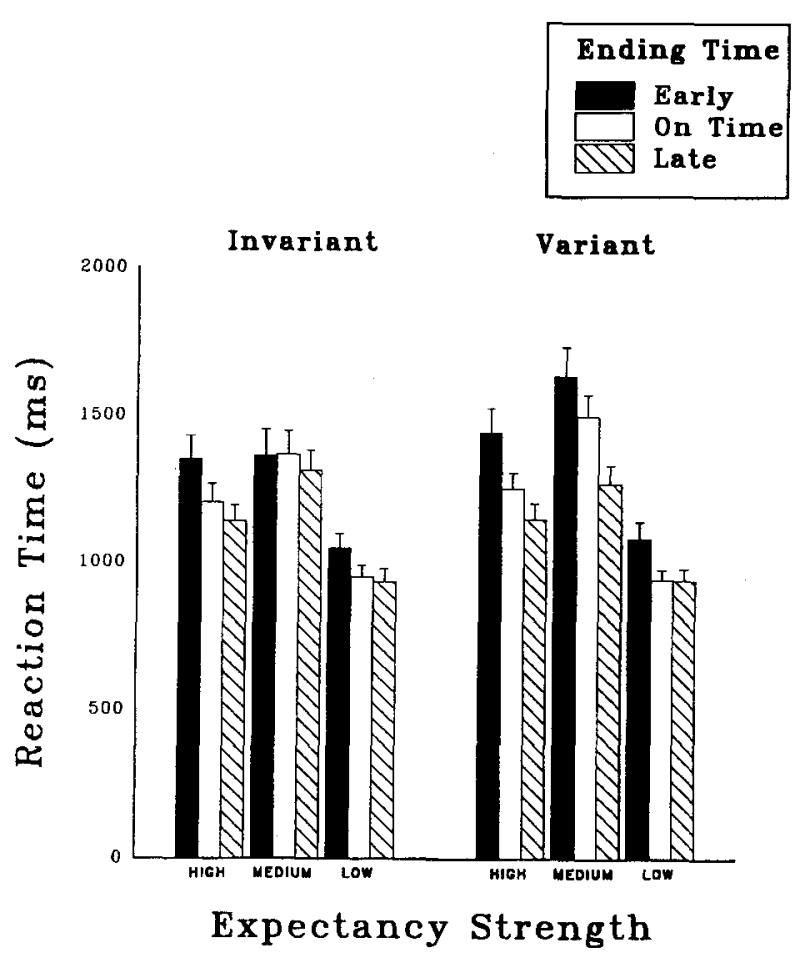

Figure 4. The mean reaction time to respond "belong" (high- and medium-expectancy chords) and "not belong" (low-expectancy chords) in Experiment 2, as a function of harmonic identity, temporal periodicity, and ending time. These reaction times are averaged across the factor of chord cadence. 
tinuations were influenced by rhythmic context and the confirmation/violation of temporal expectancies concerning the final chord of a sequence. In addition, they illustrate that both rhythmic and harmonic contexts exert an impact on the processing speed of musical information. This result is consistent with a study by Tekman and Bharucha (1992), who demonstrated that variation in the stimulus onset asynchrony between prime and target chords modulated the strength of the harmonic priming observed. Although these authors interpret these results in terms of the buildup of priming over time, one implication is that very basic temporal variation will influence harmonic expectation.

The second interesting finding of Experiment 2 is that response time varied with the harmonic appropriateness of endings; the fastest reaction times were observed for high- and low-expectancy endings, and the slowest reaction times were observed for medium-expectancy material. This is precisely what one would predict. Endings of both high- and low-expectancy strength provide obvious fits and misfits, respectively, with the underlying tonal hierarchy of the melody's key, and thereby afford fast decision times. Endings of intermediate strength, however, can potentially fall into either category-harmonic appropriateness or inappropriateness - and may therefore require more processing time to resolve their ambiguity.

\section{GENERAL DISCUSSION}

The results of these experiments confirm the predictions of models addressing the cognitive representation of musical information (Bharucha, 1987a, 1987b; Krumhansl \& Kessler, 1982). According to these approaches, the perception of tonal relationships is guided by an internal hierarchy that reflects the tonal stability of those pitch intervals within a given diatonic scale, as well as the degree of relatedness among different chords and tonal systems (i.e., keys). Thus, when listening to a musical composition, this internal hierarchy presumably dictates the course of expectancy generation so that some events will be heard as likely continuations while others will not. These predictions have, in fact, been supported by numerous experiments (Bharucha \& Stoeckig, 1986, 1987; Schmuckler, 1989) and were further evidenced here by the pattern of expectancy ratings (Experiment 1) and judgments of harmonic appropriateness (Experiment 2).

The present results, however, also suggest that such cognitive representations are not static in nature, but can be dynamically influenced by the unfolding temporal structure of a musical passage. Both Experiments 1 and 2 , for example, found that high-expectancy chords were judged as most appropriate to a melody when they unfolded with an invariant periodicity as opposed to a variant, unpredictable one. In addition, the timing of the final to-be-judged events exerted an impact in that these chords were judged as most appropriate when they appeared with the same temporal periodicity (i.e., on time) as the preceding context chords. Together, these findings suggest that the confirmation of temporal expectancies enhances the perceived expectedness of harmonic relationships. Indeed, expectancy ratings declined when the final chord occurred either one beat earlier or later than expected. Interestingly, these effects were asymmetrical-early endings had a stronger impact than late ones. One possibility is that early endings are considered particularly unexpected because the occur before their expected point of arrival and thereby catch a listener by surprise. In contrast, late endings, though unexpected because they do not occur when anticipated, do not surprise a listener, who has, after all, been kept waiting for this event. Such an interpretation suggests (indeed depends upon) a partial dissociability between musical expectancy and musical surprise - a result that has been observed in some recent work by Bradshaw (1993).

The results of the reaction time data also support this general idea. Events occurring early, relative to an expected position in time, were responded to more slowly than events occurring either on time or late; additionally, there was an insignificant (albeit suggestive) trend for late events to require less processing time than those occurring on time. Again, these differences could be a result of an interplay between surprise (resulting in slower reaction times) and expectancy (since listeners have been waiting for this event, they respond quickly). Although these suggestions are admittedly speculative, they do highlight a need for future studies that systematically investigate the relationship between surprising and expected musical events.

The interaction between tonal and temporal expectancies observed here both conforms to and extends previous findings. For example, Boltz (1989a) found that the perceived resolution of folk melodies was simultaneously influenced by both the tonal and temporal context of their endings. In particular, listeners judged the most complete endings as those containing highly expected tonal information occurring on time, relative to the preceding temporal context. Similarly, Boltz (1993) found that the detection of deviant pitch information was dependent upon the interactive influence of both temporal and pitch accent structure. The present results extend these findings by demonstrating the impact of timing variables over an extended musical passage and with respect to the harmonic (chord) structure of a passage. As such, these results generalize beyond simple resolution and/or endings of a melody.

Why does time exert this effect on musical expectancy? Some insight comes from the work of Boltz and Jones (Boltz \& Jones, 1986; Jones \& Boltz, 1989), who argue that the temporal and pitch relationships within a tune are inextricably entwined on both a structural and behavioral level. In the present experiments, chord sequences in the invariant conditions always occurred on the first, metrically strong beat of a measure (Palmer \& Krumhansl, 1991) and thereby attentionally outlined the hierarchical arrangement of melodic structure. More importantly, however, the predictable recur- 
rence of chord onsets allowed a listener to extrapolate the melody's preceding context and anticipate that future chords would appear with the same temporal periodicity. This ability to generate expectancies about the upcoming "what" and "when" of a melody both facilitates attentional tracking and reduces the amount of processing effort. The subsequent confirmation or violation of these temporal expectancies, in turn, draws attention to accompanying harmonic information and its relative status in the internal representation of the tonal relations.

Some evidence for these ideas was reflected in the present set of data. In both experiments, the highest expectancy ratings were observed when the harmonic and temporal expectancies were jointly confirmed. Conversely, expectedness decreased when the structure of a passage either failed to support the generation of temporal expectancies (i.e., variant timing) or independently violated the subsequent outcome of temporal and harmonic expectancies (i.e., early and late endings, and medium- and low-expectancy chords, respectively). These findings reinforce the notion that the joint relationship between the pitch and temporal structure of a passage exerts a corresponding influence on musical behavior.

In sum, the results of the present research suggest that current models of musical structure are incomplete without simultaneously taking into account the role of harmonic and rhythmic factors. It was noted at the outset that such influences could be of varying types. One possibility is that variation in the rhythmic structure of a passage has a dramatic effect on musical expectancies, in that an unexpected event in one context becomes an expected event in a second situation. Results along these lines call into question the very nature and form of current models addressing the cognitive representation of musical structure (e.g., Bharucha, 1987a, 1987b, 1989; Bharucha \& Todd, 1989) that assume a stable network of connections between individual tones, harmonic events (chords), and larger musical contexts (tonal centers). Such relatively permanent structures would clearly have difficulty in accounting for radical changes in expectancy formation (and hence subsequent processing) for equivalent harmonic events in varying rhythmic contexts.

An alternative possibility is that such factors have a less drastic effect on expectancy formation and processing. In this case, variation in rhythmic context modulates tonal-harmonic expectancy formation, without introducing dramatic changes in perceived expectancy. As such, one might observe subtle, consistent changes in perceived expectations across different rhythmic contexts, although the ordering of high-, medium-, and lowexpectancy events remains invariant. The results of the present experiments clearly favor this interpretation. Rather than question the structure of models of musical representations, these results imply that such models need some form of rhythmic and/or metrical weighting, so that the relative strengths of tonality, chord, and note units can be modified by the rhythmic context of an inducing passage. One conceivable instantiation of such effects lies in the rate of decay of activation throughout the networks of such models. Alternatively, it might be that explicit weighting mechanisms, encompassing metric, rhythmic, and duration information, need to be added to these systems in order to account for such variation. Unfortunately, the present results are unable to shed light on which of these alternatives is most likely.

In sum, the present experiments examined the nature of cognitive representations of musical structure by exploring general issues in musical expectancy. This work suggests that such models, which have focused on the representation of pitch, harmonic, and tonal relations, are incomplete without taking into account metrical and rhythmical factors. Future directions for this work might involve further exploration on the nature of pitch and rhythm interrelationships in expectancy formation, along with examination of the relation between expectancy formation and the processing of musical information. Such research would shed further light on the cognitive representation of musical structure and those constraints engendered by the particular unfolding context of temporal and harmonic relationships.

\section{REFERENCES}

AbE, J., \& Hoshino, E. (1990). Schema driven properties in melody cognition: Experiments on final tone extrapolation by music experts. Psychomusicology, 9, 161-172.

BERRY, W. (1976). Structural functions of music. Englewood Cliffs, NJ: Prentice-Hall.

BhARUCHA, J. J. (1987a). MUSACT: A connectionist model of musical harmony. In Proceedings of the Ninth Annual Conference of the Cognitive Science Society (pp. 508-517). Hillsdale, NJ: Erlbaum.

BharUCHA, J. J. (1987b). Music cognition and perceptual facilitation: A connectionist framework. Music Perception, 5, 1-30.

BharuCha, J. J. (1989). Pitch, harmony, and neural nets: A psychological perspective. Computer Music Journal, 13, 84-95.

BharuCha, J. J., \& KrumhansL, C. L. (1983). The representation of harmonic structure in music: Hierarchies of stability as a function of context. Cognition, 13, 63-102.

BharuCha, J. J., \& Stoeckig, K. (1986). Reaction time and musical expectancy. Journal of Experimental Psychology: Human Perception \& Performance, 12, 403-410.

Bharucha, J. J., \& Stoeckig, K. (1987). Priming of chords: Spreading activation or overlapping frequency spectra? Perception \& Psychophysics, 41, 519-524.

Bharucha, J. J., \& ToDd, P. M. (1989). Modelling the perception of tonal structure with neural nets. Computer Music Journal, 13, 128137.

Boltz, M. (1989a). Rhythm and "good endings": Effects of temporal structure on tonality judgments. Perception \& Psychophysics, 46, 917.

Boltz, M. (1989b). Time judgments of musical endings: Effects of expectancies on the "filled interval effect." Perception \& Psychophysics, 46, 409-418.

Boltz, M. (1991). Some structural determinants of melody recall. Memory \& Cognition, 19, 239-251.

Boltz, M. (1993). The generation of temporal and melodic expectancies during musical listening. Perception \& Psychophysics, 53, 585600.

Boltz, M., \& Jones, M. R. (1986). Does rule recursion make melodies easier to reproduce? If not, what does? Cognitive Psychology, 18, 389.431 
Bradshaw, D. H. (1993, June). Identification of expectancy and musical surprise in melodies. Paper presented at the annual conference of the Society for Music Perception and Cognition, Philadelphia, PA.

CARLSEN, J. C. (1981). Some factors which influence melodic expectancy. Psychomusicology, 1, 12-29.

CONE, E. (1968). Music form and musical performance. New York: Norton.

Cuddy, L., Cohen, A. J., \& Miller, J. (1979). Melody recognition: The experimental application of musical rules. Canadian Journal of Psychology, 33, 148-157.

DeuTSCH, D. (1969). Music recognition. Psychological Review, 76, 300-307.

DEUTSCH, D. (1980). The processing of structured and unstructured tonal sequences. Perception \& Psychophysics, 28, 381-389.

DEUTSCH, D., \& FEROE, J. (1981). The internal representation of pitch sequences in tonal music. Psychological Review, 88, 503-522.

Dowling, W. J. (1978). Scale and contour: Two components of a theory of memory for melodies. Psychological Review, 85, 341-354.

GJERDINGEN, R. O. (1989). Using connectionist models to explore complex musical patterns. Computer Music Journal, 13, 67-75.

GJERDINGEN, R. O. (1990). Categorization of musical patterns by selforganizing neuronlike networks. Music Perception, 7, 339-370.

JoNES, M. R. (1976). Time, our lost dimension: Toward a new theory of perception, attention and memory. Psychological Review, 83, 322-335.

JONES, M. R. (1987a). Dynamic pattern structure in music: Recent theory and research. Perception \& Psychophysics, 41, 621-634.

JONES, M. R. (1987b). Perspectives on musical time. In A. Gabrielsson (Ed.), Action and perception in rhythm and music (pp. 153-175). Stockholm: The Royal Swedish Academy of Music.

Jones, M. R., \& BolTZ, M. (1989). Dynamic attending and responses to time. Psychological Review, 96, 459-491.

JoNES, M. R., Boltz, M., \& KIDD, G. (1982). Controlled attending as a function of melodic and temporal context. Perception \& Psychophysics, 32, 211-218.

Jones, M. R., Boltz, M. G., \& KLEIN, J. M. (1993). Expected endings and judged duration. Memory and Cognition, 21, 646-665.

KRUMHANSL, C. L. (1979). The psychological representation of musical pitch in a tonal context. Cognitive Psychology, 11, 346-374.

Krumhansl, C. L. (1990). Cognitive foundations of musical pitch. Oxford: Oxford University Press.

Krumhansl, C. L., Bharucha, J. J., \& Castellano, M. A. (1982). Key distance effects on perceived harmonic structure in music. Perception \& Psychophysics, 32, 96-108.

Krumhansl, C. L., \& Kessler, E. J. (1982). Tracing the dynamic changes in perceived tonal organization in a spatial representation of musical keys. Psychological Review, 89, 334-368.

Krumhansl, C. L., \& ShePard, R. N. (1979). Quantification of the hierarchy of tonal functions within a diatonic context. Journal of Experimental Psychology: Human Perception \& Performance, 5, 579594.

LERDAHL, F., \& JACKENDOFF, R. (1983). A generative theory of tonal music. Cambridge, MA: MIT Press.

MEYER, L. B. (1956). Emotion and meaning in music. Chicago: University of Chicago Press.

MEYER, L. B. (1973). Explaining music: Essays and explorations. Chicago: University of Chicago Press.

Monahan, C., \& Carterette, E. (1985). Pitch and duration as determinants of musical space. Music Perception, 3, 1-32.

NARMOUR, E. (1989). The "genetic code" of melody: Cognitive structures generated by the implication-realization model. Contemporary Music Review, 4, 45-63.

NARMOUR, E. (1991a). The analysis and cognition of basic melodic structures. Chicago: University of Chicago Press.

NARMOUR, E. (1991b). The analysis and cognition of melodic complexity. Chicago: University of Chicago Press.

Palmer, C., \& Krumhansl, C. L. (1987a). Independent temporal and pitch structures in determination of musical phrases. Journal of Experimental Psychology: Human Perception \& Performance, 13, 116-126.
Palmer, C., \& Krumhansl, C. L. (1987b). Pitch and temporal contributions to musical phrase perception: Effects of harmony, performance timing, and familiarity. Perception \& Psychophysics, 41, 505-518.

Palmer, C., \& Krumhansl, C. L. (1991). Mental representations for musical meter. Journal of Experimental Psychology: Human Perception \& Performance, 16, 728-741.

PISTON, W. (1978). Harmony (rev. ed.). New York: Norton.

SCHMUCKLER, M. A. (1989). Expectation in music: Investigation of melodic and harmonic processes. Music Perception, 7, 109-150.

SCHMUCKLER, M. A. (1990). The performance of global expectations. Psychomusicology, 9, 122-147.

Tekman, H. G., \& BhaRucha, J. J. (1992). Time course of chord priming. Perception \& Psychophysics, 51, 33-39.

TodD, R., Boltz, M., \& JonEs, M. R. (1989). The MIDILAB auditory research system. Psychomusicology, 8, 83-96.

UNYK, A. M., \& CARLSEN, J. C. (1987). The influence of expectancy on melodic perception. Psychomusicology, 7, 3-23.

\section{NOTES}

1. These excerpts were taken from Sixty Star Hits, published by Keys-Hansen, Inc., Miami Beach, FL, 1962. Use of these excerpts conformed to the copyright laws as specified by the House Report, No. 94-1476, Section 107.

2. The sole variation on this theme was the onset of the chord occurring in the third measure, which could only occur on beats two or three, in order to avoid an overlap with the onset of the fourth and final chord.

3. Because of the various levels of expectancy strength, this comparison was run as a two-way ANOVA, with the factors of ending time (early, on-time, late) and expectancy strength (high, medium, and low expectancy). Although there was no effect for ending time (see text), there was an effect of expectancy strength $[F(2,22)=19.41, p<.001]$, but no interaction between the two $[F(4,44)=1.28$, n.s.]. The effect of expectancy strength is not surprising, given that the context melodies were occasionally modified so that they fit well with the high-expectancy condition.

4. Although these analyses demonstrate that quantifications of the melodies themselves did not differ in any systematic way, this does not necessarily mean that the listeners' ratings did not reflect these aspects of the congruence between melodic line and chord occurrence. To assess this possibility, a series of regression analyses related the listeners' ratings to the different quantifications of the melodic information in these passages, for both individual and averaged subject data. The only interesting result was a significant correlation between the averaged expectancy ratings and the number of melody tones sounding in the final chord. Similar to the results of the two-way ANOVA on this effect (see Note 3), this result is not surprising given that these melodies were constructed to fit the high-expectancy condition, suggesting that this measure is in many ways simply an index of the perceived expectancy strength. It appears, then, that expectancy ratings did not reflect, in any obvious way, properties of the melodic line occurring simultaneously with chord events.

5. If one accepts this division into correct and incorrect responses, it should then be possible to calculate the correlation between reaction times for the different sequences and the "correctness" of this response, thereby examining potential speed-accuracy tradeoffs. This analysis, however, is problematic for a variety of reasons. First, our data are subjective judgments of belongingness, and not objective correct versus incorrect responses. Although we have argued that the belong/not-belong distinction is conceptually akin to correct/incorrect, the two cannot be treated equivalently. Second, because we have used this subjective judgment, division into correct/incorrect categories mixes the actual responses of the subjects, raising questions as to the validity and subsequent interpretations of comparisons across response types. Finally, examination of Figure 3 reveals that there were, in fact, few "incorrect" responses in this experiment, particularly for the high- and low-expectancy events. Accordingly, a speed-accuracy analysis would be based on very few observations, and almost exclu- 
sively on ones for medium-expectancy chords. Based on these problems, then, the results of a speed-accuracy analysis would be, at best, inconclusive, and at worst, inappropriate and misleading.

6 . The only other notable results from this analysis were significant two-way interactions between chord cadence and harmonic identity $\left[F(4,124)=2.92, M S_{\mathrm{e}}=242,186.2, p<.05\right]$, and chord cadence and ending time $\left[F(4,124)=2.63, M S_{\mathrm{e}}=233,346.72, p<.05\right]$, as well as a significant three-way interaction between chord cadence, harmonic identity, and ending time $\left[F(8,248)=2.98, M S_{\mathrm{e}}=253,012.9\right.$, $p<.01]$. These results indicate that across all conditions of harmonic identity, reaction times in the authentic and deceptive cadence conditions were significantly longer for early endings that for those that were either on time or late. The half cadence sequences also displayed this same pattern of results for chords of both high- and lowexpectancy strength. However, reaction times for medium times were reversed in that they were longer for on-time endings than for either early or late endings.

(Manuscript received July 27, 1993;

revision accepted for publication February 18, 1994.) 